\title{
CAMPUS UND KARRIERE
}

\section{IAB-Arbeitsmarkt- barometer fängt sich auf niedrigem Niveau}

\section{Stärkster Einbruch seit Aufzeichnungsbeginn}

Das IAB-Arbeitsmarktbarometer ist im Mai gegenüber dem historischen Tiefststand im Vormonat um 0,4 Punkte gestiegen. Mit 93,8 Punkten weist der Frühindikator des Instituts für Arbeitsmarkt- und Berufsforschung (IAB) aber noch immer auf eine deutliche Verschlechterung des Arbeitsmarkts in den nächsten Monaten hin. „Der Einbruch am Arbeitsmarkt setzt sich fort, wenngleich nicht mehr mit derselben Dramatik wie in den letzten zwei Monaten. Die Talsohle ist noch nicht erreicht“", sagt Enzo Weber, Leiter des IAB-Forschungsbereichs ,Prognosen und gesamtwirtschaftliche Analysen“".

Im Mai legte die Beschäftigungskomponente des IAB-Arbeitsmarktbarometers gegenüber dem Vormonat um 0,4 Punkte auf 94,9 Punkte zu. Auch die Komponente zur Vorhersage der Arbeitslosigkeit erholte sich etwas und liegt nach einem Plus von ebenfalls 0,4 Punkten bei 92,7 Punkten. Ein Wert von unter 100 signalisiert Verschlechterungen in den drei Folgemonaten.

Die Wirtschaftskrise führt dazu, dass deutlich mehr Beschäftigungsverhältnisse beendet werden. Dennoch sei der Arbeitsmarkt vor der Covid19-Pandemie robust gewesen, erklärt Weber: „Derselbe Schock hätte vor 20 Jahren die Beschäftigung ins Bodenlose stürzen lassen. Das ist trotz der immensen Verwerfungen jetzt nicht $\mathrm{zu}$ erwarten“. Kritisch sei die eingebrochene Einstellungsbereitschaft: „Bei geringer Kapazitätsauslastung und im- menser Unsicherheit sacken die Neueinstellungen ab. Das trifft vor allem Arbeitslose und Berufseinsteiger.“

Das IAB-Arbeitsmarktbarometer ist ein seit dem November 2008 be- stehender Frühindikator, der auf einer monatlichen Umfrage der Bundesagentur für Arbeit unter allen lokalen Arbeitsagenturen basiert. Während Komponente A des Barometers die

\section{Das IAB-Arbeitsmarktbarometer im Mai 2020}

Der Arbeitsmarktausblick für die nächsten 3 Monate ist ...

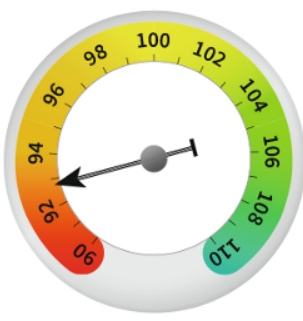

Komponente A Arbeitslosigkeit

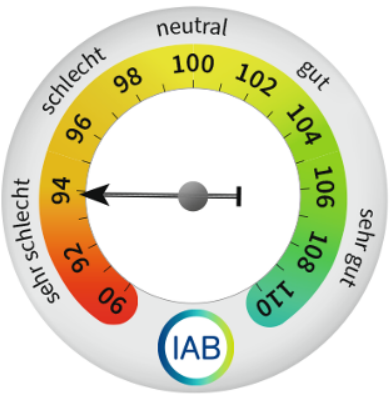

Arbeitsmarktbarometer

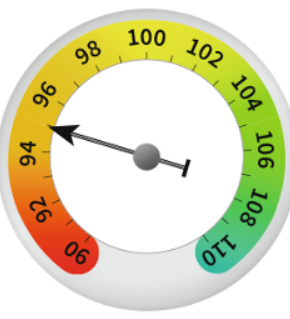

Komponente B Beschäftigung

Quellen: Berechnungen des IAB, Statistik der BA, Arbeitsmarktberichterstattung der BA. (C) IAB

Das IAB-Arbeitsmarktbarometer im Mai 2020

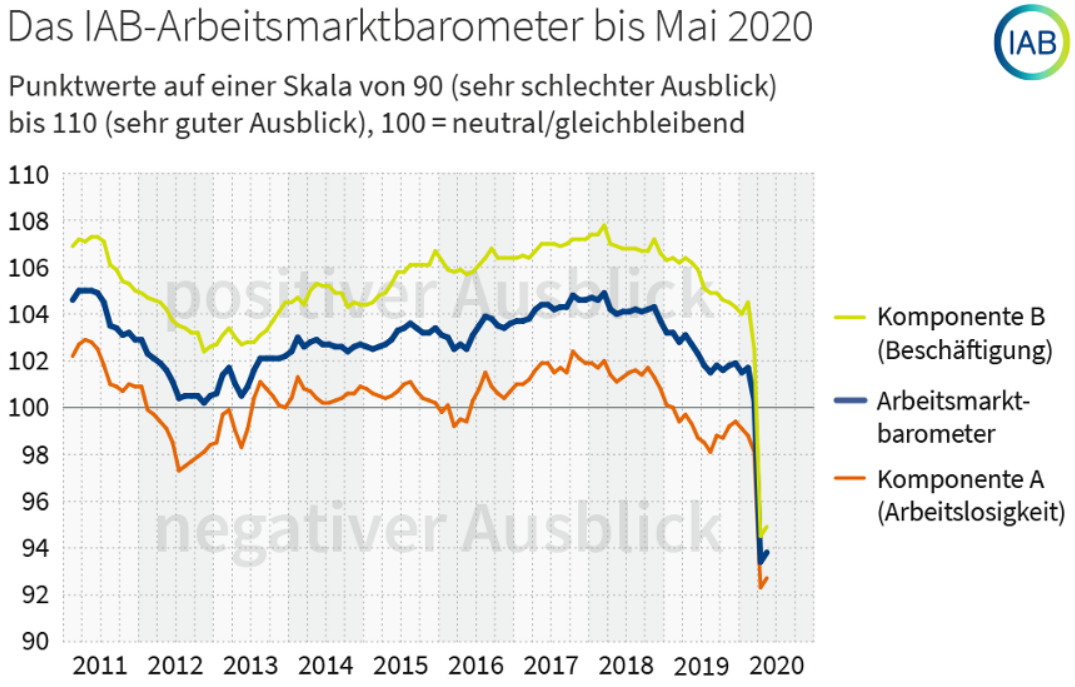

Quellen: Berechnungen des IAB, Statistik der BA, Arbeitsmarktberichterstattung der BA. (C IAB

Das IAB-Arbeitsmarktbarometer bis Mai 2020 
Entwicklung der saisonbereinigten Arbeitslosenzahlen für die nächsten drei Monate prognostiziert, dient Komponente B der Vorhersage der Beschäftigungsentwicklung. Der Mittelwert aus den Komponenten „Arbeitslosigkeit“" und „Beschäftigung“ bildet den Gesamtwert des IAB-Arbeitsmarktbarometers. Dieser Indikator gibt damit einen Ausblick auf die Gesamtentwicklung des Arbeitsmarkts. Da das Saisonbereinigungsverfahren laufend aus den Entwicklungen der Vergangenheit lernt, kann es zu nachträglichen Revisionen kommen. Die Skala des IAB-Arbeitsmarktbarometers reicht von 90 (sehr schlechte Entwicklung) bis 110 (sehr gute Entwicklung).

IAB

\section{Homeoffice in der Pandemie}

\section{Aufgabenverteilung in Familien ändern sich}

Der Lockdown zur Eindämmung von Covid-19 kann eine Verstärkung von traditionellen Rollenmustern in Familien zur Folge haben. Zwar bietet Homeoffice Müttern die Chance, ihre Arbeitszeit aufzustocken. Gleichzeitig müssen sie aber auch mehr Haushaltsund Erziehungsarbeit übernehmen vor allem, wenn die Väter nicht von zu Hause aus arbeiten können. Nur in etwa $30 \%$ der Haushalte sind Väter beruflich flexibler als Mütter und könnten daher mehr Zeit für die Kindererziehung und den Haushalt aufwenden. In der Mehrzahl der Familien kommt allerdings Mehrarbeit auf Frauen zu. Das geht aus einer aktuellen Kurzexpertise des ZEW Mannheim hervor.

„Wenn Väter jetzt mehr Haushaltsaufgaben übernehmen, können Mütter davon langfristig profitieren“, sagt Prof. Dr. Melanie Arntz, stellvertretende Leiterin des ZEW-Forschungsbereichs „Arbeitsmärkte und Perso- nalmanagement" und Mitautorin der ZEW-Kurzexpertise. „Wo durch Covid-19 traditionelle Rollenmuster Aufwind haben, dürften die Karrieren von Frauen darunter leiden.“

Die Covid-19-Pandemie hat einen massiven Wandel der Arbeitswelt mit sich gebracht. Während im Jahr 2018 knapp zwölf Prozent der Beschäftigten in Deutschland regelmäßig im Homeoffice arbeiteten, waren es im April 2020 mehr als $35 \% .26 \%$ arbeiteten sogar ausschließlich von zu Hause.

„Durch die Krise ist Homeoffice wesentlich üblicher geworden. Das wird langfristig dazu führen, dass es auch seitens der Arbeitgeber positiver beurteilt wird und häufiger verfügbar ist", sagt Melanie Arntz. „Unternehmen und ihre Beschäftigten sammeln jetzt Erfahrung mit mobilem Arbeiten und stellen fest, dass es funktioniert. Außerdem wird es in Zukunft auch leichter

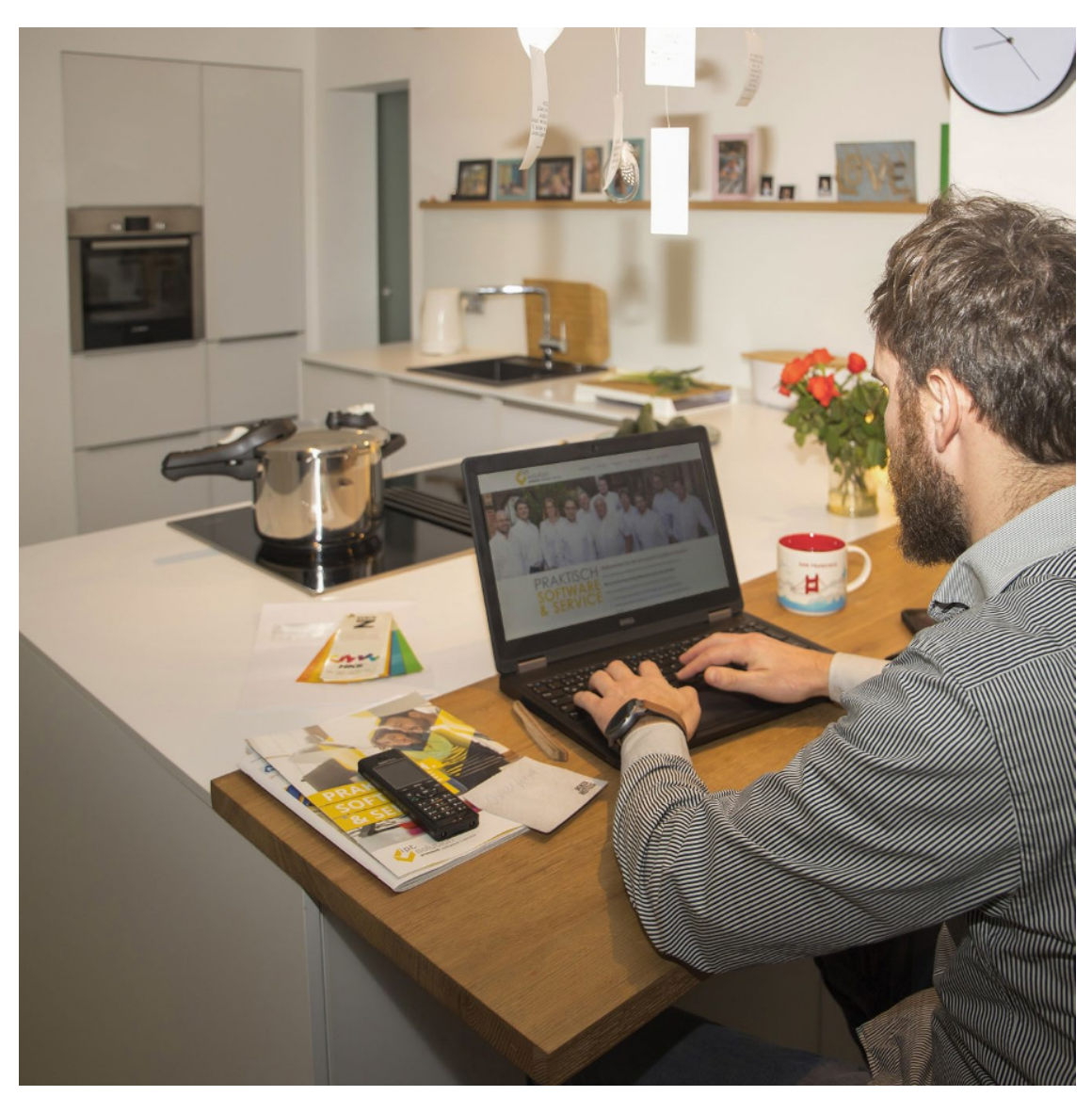

Homeoffice wird von Männern und Frauen unterschiedlich wahrgenommen und genutzt. (Foto:

Rolf Hassel/Pixabay) sein, von zu Hause aus zu arbeiten, weil die Unternehmen jetzt entsprechende Investitionen tätigen und ihre Prozesse anpassen mussten. Wahrscheinlich werden Unternehmen auch nach der Krise häufiger Arbeitsformen ermöglichen, die Tätigkeiten und Meetings vor Ort ersetzen. Wenn Homeoffice besser verfügbar ist, führt das dazu, dass insbesondere Mütter mehr arbeiten.“

Die langfristigen Wirkungen werden aber auch davon abhängen, wie die zusätzlichen Betreuungsaufgaben zwischen Müttern und Vätern verteilt werden, die aufgrund der Schul- und Kitaschließungen anfallen. Um dies $\mathrm{zu}$ untersuchen, betrachten die Wissenschaftlerinnen und Wissenschaftler, wie flexibel das Beschäftigungsver-

\section{Mütter sind in den meisten Familien beruflich flexibler}


hältnis beider Elternteile einer Familie während der Pandemie ist. Dabei gelten systemrelevante Berufe und andere Tätigkeiten, die vor Ort stattfinden müssen, als besonders unflexibel. Arbeitslosigkeit bzw. Kurzarbeit sind besonders flexibel. Begrenzt flexibel sind Berufe, die sich teilweise oder vollständig im Homeoffice erbringen lassen. Betrachtet man nun die Haushalte mit Kindern unter 13 Jahren nach der Flexibilität der beruflichen Tätigkeit der beiden Partner, so zeichnet sich ab, dass in der Mehrzahl der Familien vermutlich Mehrarbeit auf die Frauen zukommt.

In insgesamt $28 \%$ der Haushalte verfügen Mütter in der Corona-Krise über mehr Flexibilität als Väter und können deshalb mehr Erziehungs- und Haushaltsaufgaben übernehmen als zuvor. In etwa $24 \%$ der Familien ist die Flexibilität beider Eltern vergleichbar. Auch in diesen Haushalten ist es wahrscheinlich, dass Mütter mehr Zeit für Familie und Hausarbeit aufwenden werden. Denn schon vor der Pandemie war die Aufgabenverteilung in Haushalten mit Kindern unter 13 Jahren in Deutschland sehr ungleich. Selbst bei Doppelverdiener-Paaren wandten Mütter etwa dreimal so viel Zeit für die Kindererziehung und doppelt so viel Zeit für Haushaltsarbeit auf wie Väter. Bei $85 \%$ dieser Paare arbeiteten die Mütter weniger Stunden, in über $60 \%$ der Fälle verdienten sie einen geringeren Stundenlohn.

Allerdings arbeiten zurzeit $40 \%$ der Mütter, aber nur 23\% der Väter in einem während der Krise systemrelevanten Beruf. Insgesamt verfügt in knapp $30 \%$ aller Haushalte mit einem Kind unter 13 Jahren die Mutter über weniger berufliche Flexibilität als der Vater. In diesen Haushalten könnte Covid-19 also der traditionellen Rollenverteilung entgegenwirken. „Wenn Väter jetzt mehr Aufgaben in der Kindererziehung und im Haushalt übernehmen, könnte das langfristig positive Folgen für Frauen haben“, sagt Melanie Arntz. „Für einen beträchtlichen Anteil der Familien ist das jedoch nicht der Fall.
Wenn der Lockdown die klassische Rollenverteilung stärkt, profitieren Frauen von einer verstärkten Nutzung der Heimarbeit nach der Corona-Pandemie vermutlich weniger, mit nachteiligen Auswirkungen auf ihre langfristigen Karrierechancen.“

Ansprechpartner: Prof. Dr. Melanie Arntz, Stellvertretende Leiterin des ZEW-Forschungsbereichs, ,Arbeitsmärkte und Personalmanagement“, Tel: 0621/1235-159, melanie.arntz@ zew.de. Originalpublikation: Download der Kurzexpertise: http://ftp.zew.de/pub/zewdocs/ZEWKurzexpertisen/EN/ZEW_Shortreport2009.pdf.

ZEW

\section{Studierende nutzen Online- Informationen oft wenig kritisch}

\section{CORA-Studie untersucht die Fähigkeit, Internetinformationen einzuschätzen}

Das Internet und die Sozialen Medien gehören heute zu den meist genutzten Informationsquellen. Auch Studierende lernen oft lieber mit Hilfe von Online-Informationen als mit den herkömmlichen Unterrichtsmaterialien der Universitäten. Allerdings haben sie Schwierigkeiten, Informationen aus dem Internet angemessen kritisch einzuschätzen und lassen sich von unzuverlässigen Quellen beeinflussen. Dies ergab eine Studie an der Johannes Gutenberg-Universität Mainz (JGU) und der Goethe-Universität Frankfurt, bei der Studierende verschiedener Fachrichtungen wie der Medizin und der Wirtschaftswissenschaften an einem Online-Test, dem Critical Online Reasoning Assessment (CORA), teilnahmen. „Wir müssen leider feststellen, dass ein großer Teil der Studierenden sich von nicht relevanten und unzuverlässigen Informationen aus dem Internet verleiten lässt, wenn es darum geht, eine bestimmte Aufgabe zu be- antworten“, stellt Prof. Dr. Olga Zlatkin-Troitschanskaia von der JGU dazu fest. Die Studie erfolgte im Rahmen der Kooperation der Rhein-Main-Universitäten (RMU).

Lernen mit Hilfe des Internets bietet viele Möglichkeiten, allerdings auch Risiken. Es ist mittlerweile bekannt, dass im Netz nicht nur „Fake News“ verbreitet werden, sondern dass auch „Fake Science“ mit wissenschaftlich nicht korrekten Informationen zirkuliert. Die Problematik betrifft kontrovers diskutierte gesellschaftliche Themen wie aktuell die Corona-Krise, sie geht jedoch noch viel tiefer. „Eine kritische Grundhaltung allein reicht nicht aus. Vielmehr sind Kompetenzen gefragt, die es Internetnutzern ermöglichen, verlässliche von fehlerhaften und manipulativen Informationen zu unterscheiden. Für Studierende wäre es daher besonders wichtig, Online-Informationen zu hinterfragen und kritisch zu betrachten, um das eigene Fachwissen auf verlässlichen Informationen aufzubauen“, so Zlatkin-Troitschanskaia.

Um den Umgang von Studierenden mit Online-Informationen zu untersuchen, haben Olga Zlatkin-Troitschanskaia und ihr Team einen neuen Test entwickelt, der sich an dem „Civic Online Reasoning"-Assessment der amerikanischen Universität Stanford orientiert. Im Test werden den Probanden kurze Aufgaben präsentiert. Sie sollen dann frei im Internet recherchieren und sich dabei auf relevante und zuverlässige Informationen konzentrieren, um die Testfragen in der vorgegebenen, relativ kurzen Zeit von zehn Minuten zu beantworten. Die Antworten sind dabei jeweils mit Argumenten aus den genutzten Online-Informationen zu begründen.

\section{CORA-Testung erfordert komplexe Analyse und aufwändige Auswertung}

In die Auswertung der Ergebnisse geht zum einen die Antwort der Probanden auf die Testfrage ein. Zum anderen 
wird der komplette Rechercheverlauf im Internet während der Aufgabenbearbeitung aufgezeichnet, um Stärken und Schwächen beim Umgang mit Online-Informationen genau zu untersuchen. „Wir sehen, auf welchen Seiten die Studierenden recherchiert haben und welche Informationen sie benutzt haben. Die Auswertung des gesamten Bearbeitungsprozesses erfordert komplexe Analysen und ist sehr aufwändig“, sagt Olga ZlatkinTroitschanskaia. Die Untersuchungen fanden bislang in zwei verschiedenen Bundesländern statt. Dabei wurden 160 Studierende verschiedener Fachrichtungen getestet; die meisten Probanden studierten Medizin oder Wirtschaftswissenschaften und waren im ersten oder zweiten Semester.

Das Ergebnis ist ernüchternd: Fast alle Probanden hatten Schwierigkeiten mit den Aufgaben. Im Durchschnitt erreichten die Studierenden auf einer Skala von 0 bis 2 Punkten je Aufgabe nur 0,75 Punkte, die Spannweite betrug 0,50 bis 1,38 Punkte. ,Der Großteil der Studierenden zog überhaupt keine wissenschaftlichen Quellen zurate", sagt Zlatkin-Troitschanskaia mit einem Hinweis darauf, dass kein besonderes Fachwissen vorausgesetzt wurde. „Wir untersuchen immer wieder neue Gruppen von Studierenden und die Testung wird auch als Längsschnittstudie fortgesetzt. Seit Beginn der Untersuchungen vor zwei Jahren zeigen sich bislang immer wieder die gleichen Ergebnisse in den wiederholten Testungen, nämlich eine Punkteverteilung im unteren Bereich.“ Allerdings schneiden die Studierenden in den höheren Semestern etwas besser ab, als die Studierenden im ersten Studienjahr. Die Kompetenzen könnten somit im Studium gefördert werden. In den USA konnte mit gezielten Übungen ein deutlicher Kompetenzzuwachs bereits nach einigen Wochen festgestellt werden.

\section{Fähigkeiten zum kritischen Umgang mit Online- Informationen gezielt fördern}

Die Studie zeigt, dass es den meisten Studierenden nicht gelingt, in der vorgegebenen Zeit die Internetangebote richtig einzuschätzen und relevante Informationen von zuverlässigen Quellen aus dem Internet hinzuzuziehen, um die Testfragen zu beantworten. „Wie andere Studien zeigen, können Studierende die Zuverlässigkeit bekannter Medienportale und Internetangebote sehr wohl angemessen einschätzen. Darauf sollte man aufbauen und die Kompetenzen fördern, auch neue Quellen und Informationen kritisch einzuordnen und das Internet reflektiert zum Wissensaufbau zu nutzen“, so Olga Zlatkin-Troitschanskaia.

In der Forschung werden Fähigkeiten zum kritischen Umgang mit Informationen und digitalen Quellen als essenziell für das Lernen im

Item 11-3

Nehmen Sie sich ca. 10 Minuten Zeit, um diese Aufgabe zu bearbeiten. Besuchen Sie die folgende Internetseite:

https://www.gesundheit.de/krankheiten/fieber-grippe-erkaeltung/grippe/grippe-was-hilftgegen-den-virus-infekt

1. Ist es eine zuverlässige Informationsquelle über Grippe und Erkältung? Sie können jegliche Informationen auf dieser Webseite nutzen oder auch ein neues Fenster öffnen und im Internet suchen, wenn Sie möchten.

2. Begründen Sie Ihre Antwort mit Belegen aus den verwendeten Internetquellen. Bitte geben Sie die Adressen der zitierten Webseiten mit an.

Beispielaufgabe aus dem CORA-Test. (@) Wirtschaftspädagogik Uni Mainz)

21. Jahrhundert angesehen. Entsprechende Übungsaufgaben und Tests für die Studierenden gibt es aber noch wenige, besonders für den OnlineBereich. „Die RMU-Studie steht mit dem ersten Test dieser Art in Deutschland noch am Anfang", räumt ZlatkinTroitschanskaia ein. „Wir sind dabei, Lehr-Lernmaterialien und Trainings zu entwickeln und zu testen. Die Auswertung der Bearbeitungsdaten birgt dafür ein besonders hohes Potenzial, um Studierenden künftig gezielte Förderung anzubieten.“

Kontakt: Uni Mainz, Wirtschaftspädagogik, Prof. Dr. Olga Zlatkin-Troitschanskaia, 1sTroitschanskaia@uni-mainz.de.

Universität Mainz

\section{\#SemesterHack}

\section{Neue Ideen für das digitale Sommersemester}

Knapp 1000 Akteure beteiligten sich am \#SemesterHack, dem bundesweiten Online-Hackathon zur digitalen Hochschulbildung - veranstaltet vom Hochschulforum Digitalisierung (HFD), dem KI-Campus und dem Deutschen Akademischen Austauschdienst (DAAD). Ziel des Hackathons war es, innerhalb von 36 Stunden Ideen und Lösungen für die Herausforderungen des digitalen Sommersemesters zu erarbeiten. Aus rund 80 Konzepten wurden fünf Projekte ausgezeichnet.

Beim \#SemesterHack stellten sich interdisziplinäre Teams, zusammengesetzt aus Studierenden, Lehrenden, Hochschulpersonal und externen Interessierten, Challenges, um Studium und Lehre im digitalen Sommersemester 2020 zu gestalten. Teilnehmende des Online-Hackathons konnten entweder als Paten Challenges einreichen, diese als Hacker bearbeiten oder als Mentoren die Teams unterstützen. Programmierkenntnisse wurden nicht vorausgesetzt. 
Aus mehr als 80 Projekten zeichnete eine Jury, bestehend aus Vertretern der Veranstalter und der 16 Partnerhochschulen, die drei besten Projektideen aus. Folgende Projekte wurden prämiert und erhalten insgesamt 2000 Euro:

- Digital Lehren lernen - Praxiserfahrungen für Lehramtsstudierende (1. Platz): Lehramtsstudierenden soll ermöglicht werden, ein Pflichtpraktikum digital auf der Plattform „Corona-School“" durchzuführen;

- Survey ${ }^{2}$ - Research goes on(line): Community für Onlineforschung (2. Platz): ein Wegweiser für Studierende und Forschende, um die für sie passenden Tools für OnlineForschung zu finden;

- MatchUp! - Peer Support App for Students (3. Platz): eine MatchingApplikation, durch die Studierende sich vernetzen und einander unterstützen können.

Zwei Sonderpreise über jeweils 500 Euro erhalten die folgenden beiden Projekte:

- Nick-o-Meter - mit Schüttelfunktion (Sonderpreis kreativste Einreichung) gibt Hochschullehrende in Videochats Feedback: Per Gestenerkennung erkennt die Software, ob Studierende nicken, den Kopf schütteln oder die Hand heben;

- WIR_tuell - Wie man im digitalen Studium zusammenwächst (Sonderpreis beste Handreichung) liefert ein Handbuch dazu, wie man auch im digitalen Klassenzimmer den Aufbau sozialer Bindungen fördern kann.

\section{Vielseitige Projektvorschläge und -ideen}

Auch die weiteren eingereichten Projektideen sind ausgesprochen vielseitig und reichen von Autorentools für die Erstellung von offenen Bildungsinhalten über internationale Austauschplattformen bis hin zur KI-gestützten Studienberatung.
„Wir sind begeistert, wie viele großartigen Ideen in nur 36 Stunden \#SemesterHack entstanden sind“, resümiert Florian Rampelt, Projektleiter des KI-Campus und stellvertretender Leiter der Geschäftsstelle des Hochschulforums Digitalisierung.

„Wer behauptet, Emotionen ließen sich schlecht über das Internet transportieren, der wurde beim Hackathon eines Besseren belehrt“, fügt Alexander Knoth, Referatsleiter für Digitalisierung beim DAAD hinzu. „Die grenzüberschreitende Zusammenarbeit war ein kreatives Gemeinschaftserlebnis mit einer spürbaren Dynamik in den Teams. Der \#SemesterHack zeigt, dass sich Dinge produktiv bewegen lassen, wenn man sie nur anpackt.“

Der \#SemesterHack am 6. und 7. Mai 2020 diente gleichzeitig als Pre-Hackathon für einen globalen Hackathon im Wintersemester 2020. Das Hochschulforum Digitalisierung und der DAAD planen gemeinsam mit der Europäischen Kommission, dem EIT Climate-KIC und der Universität Aalto im November 2020 den \#DigiEduHack mit einem globalen Fokus auf Innovationen in der Bildungslandschaft. Hier sollen auch Ideen aus dem \#SemesterHack aufgegriffen und weiterentwickelt werden.

Der Online-Hackathon ergänzt die bestehenden Aktivitäten des Hochschulforums Digitalisierung und des KICampus zur Unterstützung der Hochschulen anlässlich der Corona-Krise.

Das Hochschulforum Digitalisierung orchestriert den Diskurs zur Hochschulbildung im digitalen Zeitalter. Gefördert wird es vom Bundesministerium für Bildung und Forschung (BMBF). Informationen: http://www.hochschulforumdigitalisierung.de. „KI-Campus Die Lernplattform für Künstliche Intelligenz“ ist ein vom Bundesministerium für Bildung und Forschung (BMBF) gefördertes Pilotprojekt. Im Zentrum steht der prototypische Aufbau einer digitalen Lernplattform, die Studierenden, Berufstätigen und anderen lebenslang Lernenden KI-Kompetenzen vermittelt. Informationen: http://www.ki-campus.org.

Stifterverband

\section{Wie wir in Zukunft arbeiten}

\section{IzR-Heft „Neue Arbeitswelten“ erschienen}

Wer heute erwerbstätig ist, nimmt wahr, dass sich die Arbeit verändert - und mit ihr der Raum. Die Corona-Krise beschleunigt diesen Wandel. Mehr denn je gilt, dass sich Bereiche wie Arbeiten, Wohnen, Mobilität, Betreuung, Bildung oder Digitalisierung nicht voneinander trennen lassen. Das aktuelle Heft der vom Bundesinstitut für Bau-, Stadt- und Raumforschung (BBSR) herausgegebenen Fachzeitschrift „Informationen zur Raumentwicklung“ (IzR) zeigt: Der Wandel vollzieht sich regional und branchenspezifisch unterschiedlich. Wie können Politik, Wirtschaft und Verwaltung ihn gestalten?

Die Analysen, Kommentare und Interviews im Heft beschäftigen sich mit den vielschichtigen Entwicklungen. Die Autoren zeigen, welche neuen Arbeitsformen die Digitalisierung schafft und wie Coworking-Spaces auf dem Land das Pendeln verringern. Sie gehen auf zahlreiche weitere Fragen ein: Was passiert mit Erwerbstätigen, die wegen ihrer Kinder nicht in vollem Umfang erwerbstätig sein können? Welche Rolle spielen Mittelstädte für die Kultur- und Kreativwirtschaft? Und wie integrieren Städte Büros und Industrie in Quartiere, um Arbeit und Privatleben besser miteinander zu verschmelzen?

Das Heft mit dem Titel „Neue Arbeitswelten“ ist im Franz Steiner Verlag erschienen. Interessierte können die Print-Version oder das eJournal für 19 Euro bestellen: service@ steinerverlag.de. http://www.bbsr.bund.de/BBSR/DE/ Veroeffentlichungen/IzR/izr_node.html

BBSR

Hinweis des Verlags Der Verlag bleibt in Hinblick auf geografische Zuordnungen und Gebietsbezeichnungen in veröffentlichten Karten und Institutsadressen neutral. 